\title{
Linkage disequilibrium mapping of the gene for Hermansky-Pudlak syndrome to chromosome 10q23.1-q23.3
}

\author{
Kazuyoshi Fukai ${ }^{1}$, Jangsuk Oh', Edgar Frenk ${ }^{3}$, Carmelo Almodóvar ${ }^{4}$ and Richard A.Spritz ${ }^{1,2, \star}$ \\ Departments of ${ }^{1}$ Medical Genetics and ${ }^{2}$ Pediatrics, University of Wisconsin, Madison, Wisconsin 53706, USA, ${ }^{3}$ Service de dermato- \\ venereologie, Centre Hospitalier Universitaire Vaudois, Lausanne, Switzerland and ${ }^{4}$ National Organization for Albinism and Hypopigmentation- \\ Puerto Rico, Inc., Arecibo, Puerto Rico 00612, USA
}

Received July 10, 1995; Revised and Accepted July 17, 1995

\begin{abstract}
Hermansky-Pudlak syndrome (HPS) is an autosomal recessive disorder characterized by the triad of tyrosinase-positive oculocutaneous albinism, bleeding diathesis due to storage-pool deficiency of platelets, and a lysosomal ceroid storage disease. The disorder is particularly frequent in Puerto Rico and in an isolated village in the Swiss Alps. We have used a linkage disequilibrium mapping approach to localize the HPS gene in both of these groups to a $\mathbf{0 . 6}$ centiMorgan interval in chromosome segment 10q23.1-q23.3. These results indicate that the Puerto Rican and Swiss forms of HPS are either allelic or that they result from mutations in very closely linked genes in this region. This region of distal chromosome $10 q$ is syntenic to the region of mouse chromosome 19 that includes 'pale ear' (ep) and 'ruby-eye' (ru), which must be considered as potential murine homologues to human HPS.
\end{abstract}

\section{INTRODUCTION}

Hermansky-Pudlak syndrome (HPS) is an autosomal recessive disorder characterized by the triad of tyrosinase-positive oculocutaneous albinism (OCA), a bleeding diathesis, and an apparent lysosomal ceroid storage disease (1). Other symptoms, including progressive restrictive lung disease, cardiomyopathy, renal failure, and a granulomatous enteropathy resembling Crohn's disease, occur frequently. The clinical manifestations of HPS can be extremely variable, both between and even within affected sibships. There is no specific or effective therapy for HPS, and treatment is usually limited to supportive care. Average survival is 30-50 years or less, and death usually results from restrictive lung disease $(68 \%)$, hemorrhage $(17 \%)$, or granulomatous colitis (15\%) (2). HPS is relatively rare in most human populations, but is quite prevalent in Puerto Rico, particularly in the northwestern quarter of the island, where it occurs with an estimated frequency of approximately 1 per 1800 persons (2). HPS is also very frequent in a long-isolated village in the Swiss Alps $(3,4)$, although these patients lack manifestations of ceroid storage and usually have a milder clinical course with normal life expectancy.
The fundamental defect in HPS is not known, but it is thought to involve a protein required in common for the normal function and/or structure of three different organelles: melanosomes, platelet dense granules, and lysosomes. Albinism in HPS results from a greatly reduced number of fully melanized melanosomes in skin and hairbulb melanocytes, and the melanosomes that are present exhibit reduced melanin content and abnormal internal structure (5), size, and morphology $(4,6)$. Bleeding in HPS results from defective platelet ADP release and aggregation $(7,8)$ due to a reduced number of platelet 'dense granules' $(9,10)$, storage pool granules rich in serotonin, calcium, ADP, ATP, and pyrophosphate (11). Lysosomal storage in HPS is characterized by the accumulation of yellow-brown ceroid/lipofuscin-like material in lysosomal structures of reticuloendothelial cells (1), lung macrophages (12), gastrointestinal mucosal cells (2), and cells of many other tissues. In the mouse there are 14 distinct loci in which mutations result in hypopigmentation associated with platelet and/or lysosomal defects (13-15), and several of these have been suggested as possible homologues to human HPS (1619).

We have used the approach of linkage disequilibrium mapping to localize the human HPS gene to a 0.6 centiMorgan (cM) interval of chromosome segment 10q23.1-q23.3. First proposed by Lander and Botstein in 1986 (20), linkage disequilibrium mapping is particularly well suited to the precise localization of genes for rare autosomal recessive diseases by taking advantage of identity by descent (21). In the case of HPS, the very high frequency of the disorder in the northwestern part of the island of Puerto Rico probably resulted from occult inbreeding following descent from a common founder. Similarly, the Swiss HPS kindred derives from one small isolated Alpine village, and extensive inbreeding has been described $(3,4)$. Within each group, the HPS locus should therefore be in linkage disequilibrium with closely linked marker alleles or marker allele haplotypes derived from the founder chromosome. The very large number of meioses since introduction of the founder chromosome into the population ensures a very high resolution for genetic mapping purposes. Because of the considerable differences in disease phenotype between Puerto Rican and Swiss HPS, we did not expect that these disorders would necessarily prove identical or even be allelic. However, the Puerto Rican and Swiss subtypes of HPS 
both map to precisely the same interval in 10q23.1-q23.3, indicating that either these disorders are allelic or they result from mutations in very closely linked genes in this chromosomal region. This region of human chromosome 10 is syntenic to that portion of mouse chromosome 19 that includes 'pale ear' (ep) and 'ruby-eye' ( $r u$ ) (22), suggesting that one or both of these mouse mutations may be homologous to human HPS.

\section{RESULTS}

Markers were selected to provide generally uniform and progressively denser coverage of the genome. The first 198

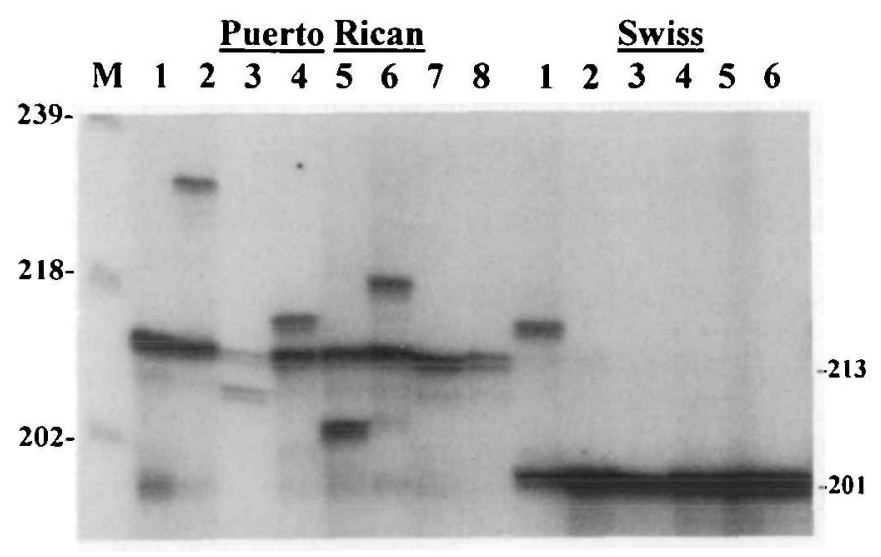

Figure 1. Genotype analysis of D $10 S 677$ in patients with HPS. Lane M, molecular size standard. Lanes 1-8, Puerto Rican HPS patients 1-8. Lanes 9-14, Swiss HPS patients 1-6. markers tested, variably distributed over all 22 autosomes, showed no evidence of linkage disequilibrium with HPS. However, marker D10S677 exhibited striking linkage disequilibrium both in the Puerto Rican and in the Swiss HPS groups. As shown in Figure 1, all eight Puerto Rican HPS probands shared the $213 \mathrm{bp}$ allele 1 of D10S677, and three were homozygous for this allele. Similarly, all six affected members of the Swiss HPS kindred shared the 201 bp allele 2 of D10S677, and five were homozygous for this allele. The probability of obtaining 11 of 16 independent chromosomes in the Puerto Rican group sharing one allele at any of 199 markers on the basis of chance is approximately 0.285 (modeled assuming nine alleles for each marker with the allele frequencies as reported for D10S677; ref. 23). Furthermore, we also determined the D10\$677 genotype for all 32 members of the Swiss HPS kindred, and by genetic linkage analysis calculated a maximum lod score of 1.99 at $\theta=0.00$ (Table 1). Although not definitive, together these results suggested both that a HPS gene might be located near D10S677 and also that the Puerto Rican and Swiss HPS patients might have mutations at the same locus on 10q, or at least at closely-linked loci.

To confirm the possible linkage of D10S677 to one or more human HPS loci, and to further localize the HPS gene, we genotyped the eight Puerto Rican HPS probands and the six affected Swiss HPS patients for 11 additional polymorphic markers in the close vicinity of D10S677 (23) (Fig. 2). For most of these markers all eight Puerto Rican patients shared at least one common allele and multiple individuals were homozygous for this allele. Similar data were observed for the six Swiss HPS patients. For some of the markers, maximum lod scores in the Swiss kindred were well in excess of 3 (Table 1).

Table 1. Two point lod scores for HPS and chromosome 10q markers in the Swiss kindred

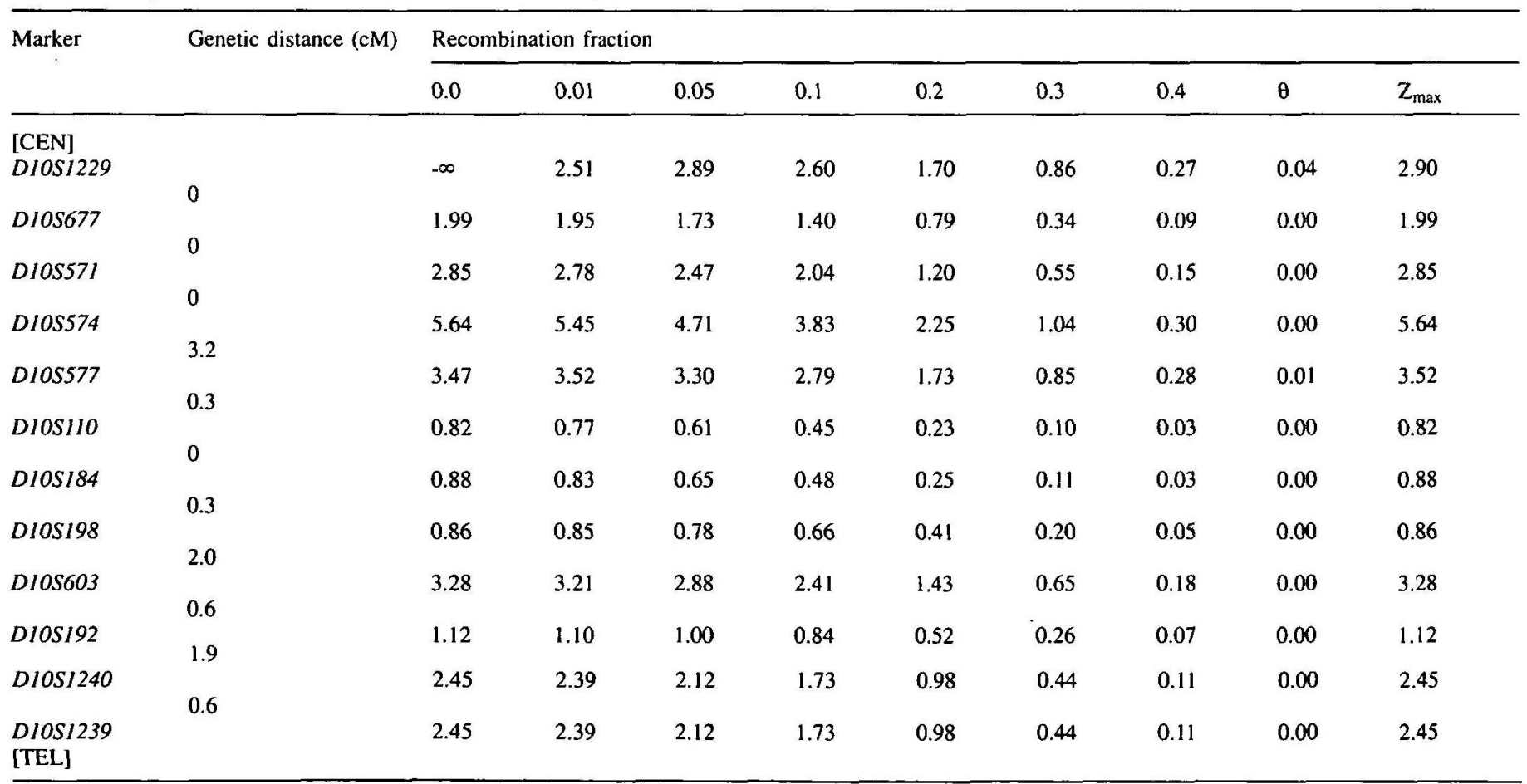

To reduce computational time, the highly inbred Swiss HPS kindred was split into four sub-families, most likely greatly reducing maximum lod scores and somewhat reducing the maximum likelihood estimates of $\theta$. 
Human Molecular Genetics, 1995, Vol. 4, No. $9 \quad 1667$

A

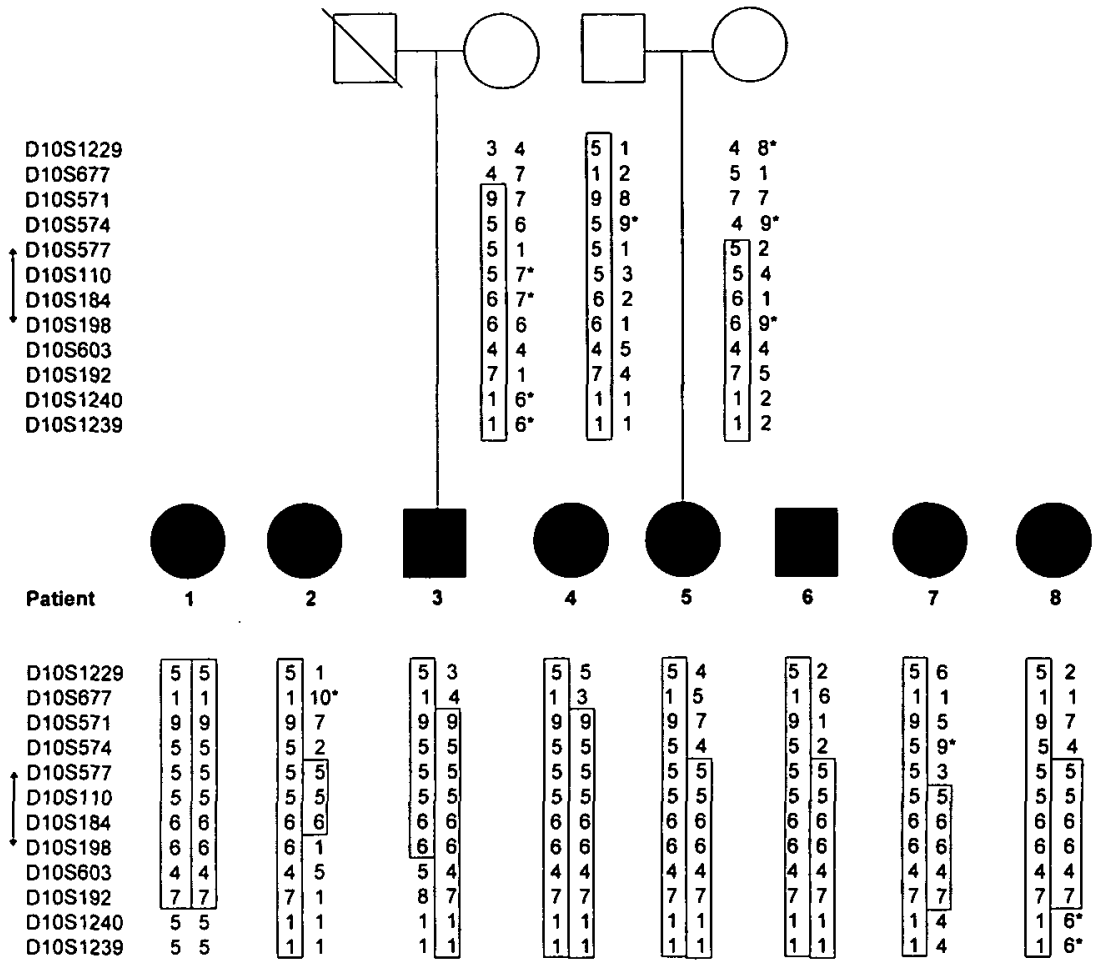

$\mathbf{B}$

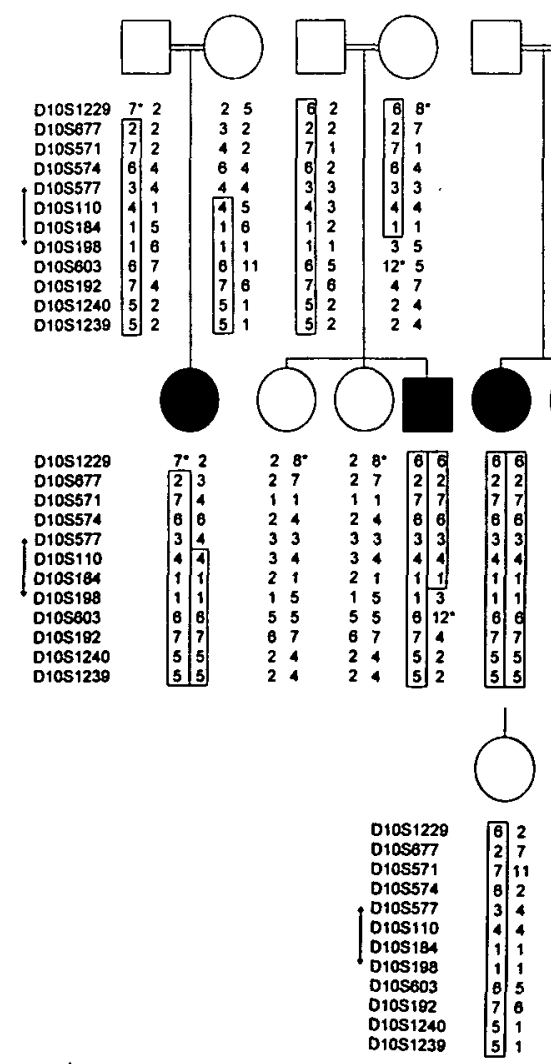

Figure 2. Chromosome 10q marker haplotypes in Puerto Rican and Swiss HPS patients and their immediate families. (A) Puerto Rican. (B) Swiss. Haplotypes or portions of haplotypes inferred as being ancestrally related to HPS are boxed. Alleles are as described in ref. 21, except for novel alleles, which are indicated by asterisks. Double-headed arrows denote the most likely interval for the HPS locus. Detailed pedigrees of the extremely large and complex Swiss HPS kindred have been reported elsewhere $(3,4)$; the individuals shown in generation I are all related to virtually all of each other. Numerous additional individuals in the Swiss HPS kindred were studied for the lod score analyses in Table 1. 
A

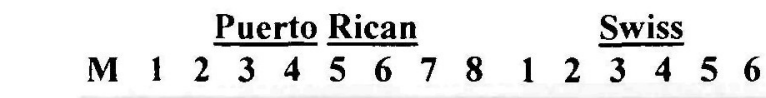

202-

181 .

B

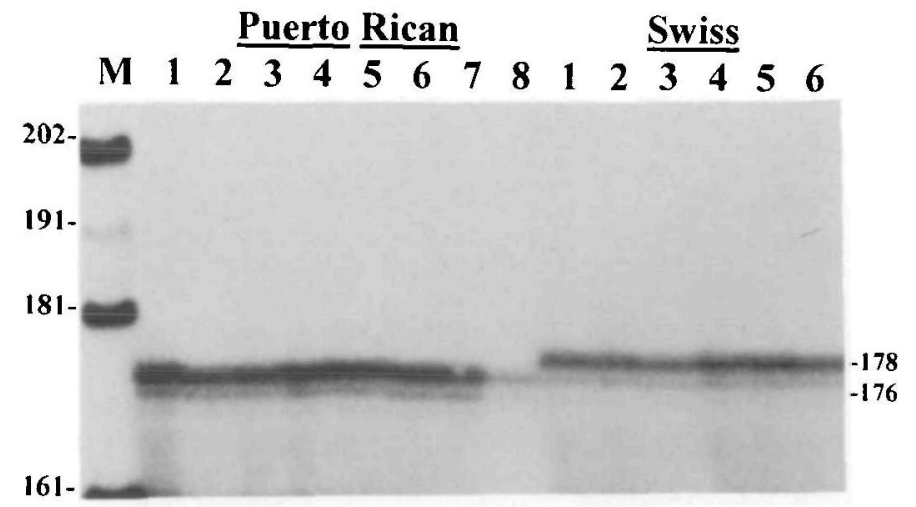

Figure 3. Genotype analysis of D10S110/D10S184 in patients with HPS. (A) DIOS110. (B) DIOS184. Lanes are as described in Figure 1.

Two markers, D10S110 and D10S184, located near the middle of this region (23), were homozygous for one allele in all eight Puerto Rican HPS probands and for a different allele in all six Swiss HPS patients (Figs 2 and 3). The probability of homozygosity for any single allele of D10S110 in eight unrelated Puerto Rican individuals on the basis of chance is $2.8 \times 10^{-4}$, and the maximum lod score for DIOS 110 in the Swiss kindred was 0.82 at $\theta=0$. The probability of homozygosity for any single allele of D10S184 in eight unrelated Puerto Rican individuals by chance is $9.4 \times 10^{-5}$, and the maximum lod score in the Swiss kindred was 0.88 at $\theta=0$. However, analysis of the primer sequences for D10S110 and D10S184, which map $0 \mathrm{cM}$ from each other (23), suggest that they most likely define the same polymorphic microsatellite; therefore, these markers should probably not be considered independent.

As shown in Figure 2, apparent ancestral marker haplotypes associated with HPS were easily derived in both the Puerto Rican and Swiss HPS patients. The frequency of apparent recombination events with HPS tended to increase in both directions from the completely homozygous marker D10S110/ D10S184. In both the Puerto Rican and Swiss HPS patients recombinants were observed between D10S110/D10S184 and D10S577 or D10S198, which flank D10S110/DI0S184 0.3
$\mathrm{cM}$ to the centromeric and telomeric sides, respectively. These data thus strongly indicate that the Puerto Rican and Swiss forms of HPS are either allelic or involve very closely linked loci situated within the $0.6 \mathrm{cM}$ interval defined by DI0S577 and D10S198, probably close to D10S110/D10S184.

\section{DISCUSSION}

We have mapped the HPS locus to a $0.6 \mathrm{cM}$ interval flanked by D10S577 and DIOS 198 and centered on DIOS110/DIOS184. D10S110 has been mapped by FISH to chromosome segment 10q23.1-q23.3 (24), establishing this chromosome segment as the cytogenetic location of the human HPS locus. Despite evident phenotypic differences between the Puerto Rican and the Swiss forms of HPS, we found no evidence for genetic heterogeneity; both mapped to this same location. Nevertheless, we cannot exclude the possibility that these two forms of the disorder result from defects in closely linked genes, perhaps members of a gene family. This is not a purely formal distinction, as two of the 14 different mouse loci associated with HPS-like recessive mutant phenotypes are located very close together in a region of mouse chromosome 19 that is syntenic to this region of distal human chromosome $10 \mathrm{q}(22)$. These two loci, 'pale ear' $(e p)$ and 'ruby-eye' $(r u)$, are both associated with reduced coat and eye pigment, misrouting of optic tract neurons, abnormal melanosomes, abnormalities of lysosomal enzymes, and deficiency of platelet dense granules and prolonged bleeding time; phenotypes very similar to human HPS. Although $e p$ and $r u$ are each referenced by only one mutant allele $(13,14)$, these two loci appear to be nonallelic (25), and map about $2 \mathrm{cM}$ apart (map positions 42 and 44, respectively), between $T d t$ and Cyp 17 (26). Neither ep nor $r u$ has been cloned, and thus neither has been mapped precisely relative to the human genome. However, human TDT maps to 10q23-q24, and human CYPI7 to $10 \mathrm{q} 24.3$ (27), coincident with the most likely location of the human HPS gene. Therefore, both $e p$ and $r u$ must be considered as potential murine homologues to human HPS.

No known human genes or ESTs have been mapped into this $0.6 \mathrm{cM}$ interval on $10 \mathrm{q}$. It thus seems probable that a positional cloning approach will be required for the eventual isolation of the HPS gene itself. Nevertheless, our precise localization of the HPS gene will permit an immediate improvement in the accuracy of genetic counseling of families in the Swiss and Puerto Rican groups at high risk for HPS. Ultimately, identification of the HPS gene should facilitate definition of its function, greatly enhancing future efforts to develop specific effective therapeutic modalities for HPS, both pharmacologic and DNA-based.

\section{MATERIALS AND METHODS}

\section{Patients}

Blood samples for DNA analysis were collected from 32 members of the Swiss HPS kindred $(3,4)$, including six affected individuals in four nuclear families. All individuals were examined by one of the authors. Blood samples for DNA analysis were collected from members of 35 unrelated Puerto Rican HPS nuclear families, including 40 affected individuals. Many had previously been studied from a clinical standpoint (2) and tested positive for HPS by lack of platelet dense granules. In none of these families were the parents knowingly consanguineous, although all families came from northwestern Puerto Rico, principally from the towns of Arecibo, San Sebastian, and Moca. All individuals were examined by at least one of the authors. Eight unrelated 
probands with unequivocal clinical findings of HPS were selected for mapping studies.

\section{Genotype analysis}

High-molecular weight DNA was prepared and genotyped using a panel of 363 polymorphic microsatellite PCR markers (Human Screening Set Version 5; Research Genetics, Inc.) by standard procedures (28). For each marker pooled DNAs of three unrelated normal caucasians were genotyped in parallel as a control. One primer of each pair was end-radiolabeled with ${ }^{32}[\mathrm{P}]$ and the PCR products were analyzed by denaturing gel electrophoresis and autoradiography. In most cases two different markers that yielded quite disparately-sized PCR products were amplified and analyzed in multiplex. Scoring was by visual inspection, and allele sizes were assigned by comparison to ${ }^{32}[\mathrm{P}]$-radiolabeled DNA molecular size standards also on the gel and by parallel analyses of CEPH pedigree members 1331-01 and 1331-02 as standards. Markers in the region of D10S677 were identified from public databases (23), and corresponding PCR primer pairs were synthesized or purchased from Research Genetics, Inc. and used to genotype the Puerto Rican HPS patients, some of their parents, and all available members of the Swiss HPS kindred as above.

\section{Linkage analysis}

Pairwise lod scores were calculated for the Swiss HPS kindred using the MLINK and ILINK programs of the LINKAGE package (Ver. 5.1) (29). The disease was considered to be autosomal recessive with a frequency of $10^{-5}$. Allele frequencies for the markers were as reported in public databases (23), except for DIOS110 and DI0S184, for which we determined allele frequencies by genotyping eight unrelated normal Puerto Rican individuals as described above.

\section{ACKNOWLEDGMENTS}

We thank C.Perez Dendariarena for help in organizing collection of patient samples in Puerto Rico and C.Denniston for statistical assistance. This work was supported by Clinical Research Grant 6-0281 from the March of Dimes Birth Defects Foundation and Grant AR39892 from the National Institutes of Health. This is paper number 3440 from the Laboratory of Genetics, University of Wisconsin.

\section{REFERENCES}

1. Hermansky, F. and Pudlak, P. (1959) Albinism associated with hemorrhagic diathesis and unusual pigmented reticular cells in the bone marrow: Report of two cases with histochemical studies. Blood 14, 162-169.

2. Witkop, C.J., Babcock, M.N., Rao, G.H.R., Gaudier, F., Sommers, C.G., Shanahan, F., Harmon, K.R., Townsend, D., Sedano, H.O., King, R.A., Cal, S.X., and White, J.G. (1990) Albinism and Hermansky-Pudlak syndrome in Puerto Rico. Bol. Assoc. Med. P. Rico 82, 333-339.

3. Lattion, F., Schneider, P., Da Prada, M., Lorez, H.P., Richards, J.G., Picotti, G.B., and Frenck, E. (1983) Syndrome d'Hermansky-Pudlak dans un village valaisan. Helv. Paediatr. Acta 38, 495-512.

4. Schallreuter, K.U., Frenk, E., Wolfe, L.S., Witkop, C.J., and Wood, J.M (1993) Hermansky-Pudlak syndrome in a Swiss population. Dermatology 187, 248-256.

5. Witkop Jr., C.J.,Quevedo, W.C., Fitzpatrick, T.B., and King, R.A. (1989) In Scriver, C.R., Beaudet, A.L., Sly, W.S., and Valle, D. (eds) The Metabolic Basis of Inherited Disease. New York, McGraw-Hill, pp. 2905-2947.

6. Frenk, E. and Lattion, F. (1982) The melanin pigmentary disorder in a family with Hermansky-Pudlak syndrome. J. Investig. Dermatol. 78, 141-143.

7. Logan, L.J., Rapaport, S.I., and Maher, I. (1979) Albinism and abnormal platelet function. N. Engl. J. Med. 284, 1340-1345.

8. Holmsen, H. and Weiss, H.J. (1972) Secretable storage pool in platelets. Blood 39, 197-209.

9. White, J.G., Edson, J.R., Desnick, S.J., and Witkop Jr., C.J. (1971) Studies of platelets in a variant of the Hermansky-Pudlak syndrome. Am. J. Pathol. 63, 319-332.

10. Hardisty, R.M., Mills, C.B., and Ketsa-Ard, K. (1972) The platelet defect associated with albinism. Br. J. Haematol. 23, 679-692.

11. Holmsen, H. and Weiss, H.J. (1979) Further evidence for a deficient storage pool of adenine nucleotides in platelets from some patients with thrombocytopathia 'storage pool disease.' Annu. Rev. Med. 30, 119-134.
12. Harmon, K.R., Witkop, C.J., White, J.G., King, R.A., Peterson, M., Moore, D., Tashjian, J., Marinelli, W.A., and Bitterman, P.B. (1994) Pathogenesis of pulmonary fibrosis: Platelet derived growth factor precedes structural alterations in the Hermansky-Pudlak syndrome. J. Lab. Clin. Med. 123, 617-627.

13. Silvers, W.K. (1979) The Coar Colors of Mice. New York, Springer-Verlag.

14. Lyon, M. and Searle, A.G. (1989) Genetic Variants and Strains of the Laboratory Mouse. Oxford University Press.

15. Bennett, D. (1991) Color genes, oncogenes, and melanocyte differentiation. J. Cell Sci. 98, 135-139.

16. Swank, R.T., Reddington, M., Howlett, O., and Novak, E.K. (1991) Platelet storage pool deficiency associated with inherited abnormalities of the inner ear in the mouse pigment mutants muted and mocha. Blood 78, 2036-2044.

17. Swank, R.T., Sweet, H.O., Davisson, M.T., Reddington, M., and Novak, E.K. (1991) Sandy: a new mouse model for platelet storage pool deficiency. Genet. Res., Camb. 58, 51-62.

18. White, R.A., Peters, L.L., Adkison, L.R., Korsgren, C., Cohen, C.M., and Lux, S.E. (1992) The murine pallid mutation is a platelet storage pool disease associated with the protein 4.2 (pallidin) gene. Nature Genet. 2 , $80-83$.

19. Spritz, R.A. and Hearing Jr., V.J. (1995) Genetic disorders of pigmentation. Adv. Hum. Genet. 22, 1-45.

20. Lander, E.S. and Botstein, D. (1986) Mapping complex traits in humans: new methods using a complete RFLP linkage map. Cold Spring Harbor Symp. Quant. Biol. 51, 49-52.

21. Houwen, R.H.K., Baharloo, S., Blankenship, K., Raeymaekers, P., Juyn, J., Sandkuijl, L.A., and Freimer, N.B. (1994) Genomic screening by searching for shared segments: mapping a gene for benign recurrent intrahepatic cholestasis. Nature Genet. 8, 380-386.

22. Poirer, C. and Guénet, J.-L. (1994) Mouse chromosome 19. Mammalian Genome 5, S266-S275.

23. Murray, J.C., Buetow, K.H., Weber, J.L., Ludwigsen, S., ScherpbierHeddema, T., Manion, F., Quillen, J., Sheffield, V.C., Sunden, S., Duyk, G.M., Weissenbach, J., Gyapay, G., Dib, C., Morrissette, J., Lathrop, G.M., Vignal, A., White, R., Matsunami, N., Gerken, S., Melis, R., Albertsen, H., Plaetke, R., Odelberg, S., Ward, D., Dausset, J., Cohen, D., and Cann, H. (1994) A comprehensive human linkage map with centimorgan density. Science 265, 2049-2054.

24. Moir, D.T., Dorman, T.E., Day, J.C., Ma, N.S.-F., Wang, M.-T., and Mao, J.-I. (1994) Toward a physical map of human chromosome 10 : Isolation of 183 YACs representing 80 loci and regional assignment of 94 YACs by fluorescence in situ hybridization. Genomics 22, 1-12.

25. Lane, P.W. and Green, E.L. (1967) Pale ear and light ear in the house mouse. Mimic mutations in linkage groups XII and XVII. J. Hered. 58, 17-20.

26. Keller, S.A., Jones, J.M., Boyle, A., Barrow, L.L., Killen, P.D., Green, D.G., Kapousta, N.V., Hitchcock, P.F., Swank, R.T., and Meisler, M.H. (1994) Kidney and retinal defects $(K r d)$, a transgene-induced mutation with a deletion of the mouse chromosome 19 that includes the Pax2 locus. Genomics 23, 309-320.

27. Online Mendelian Inheritance in Man, OMIM (TM) (database online) (1995) Baltimore, Johns Hopkins University.

28. Dracopoli, N.C., Haines, J.L., Korf, B.R., Moir, D.T., Monton, C.C., Seidman, C.E., Seidman, J.G., and Smith, D.R. (eds) (1994) Current Protocols in Human Genetics, New York, John Wiley and Sons, vol 1, pp. 2.5.1-2.5.4.

29. Terwilliger, J.D. and Ott, J. (1994) Handbook of Human Genetic Linkage. Baltimore, Johns Hopkins Press. 